\title{
Studies on Intramitochondrial Inclusions in the Pancreatic Acinar Cells of the Japanese Newt, Triturus pyrrogaster. II. The disappearance of Intramitochondrial Inclusions in Re-fed Animals following: Starvation
}

\author{
By \\ Kazuo TAIRA and Susumu SHIBASAKI \\ Department of Anatomy, Gunma University School of Medicine, \\ Maebashi 371, Japan \\ -Received for Publication, January 30, 1982-
}

\begin{abstract}
Key words: Intramitochondrial inclusions, Pancreas, Re-feeding, Newt.
Summary: Intramitochondrial inclusions in pancreatic acinar cells of the re-fed Japanese newt, Triturus pyrrogaster, following starvation were examined with the aide of an electron microscope. Clusters of mitochondria and the close apposition between mitochondria and rough endoplasmic reticulum were observed in the first few hours after re-feeding. Unusual mitochondria, which were observed in the acinar cells of the starved animals, decreased in number and size after refeeding and finally disappeared. We suggest that intramitochondrial inclusions are temporarily induced as a result of depressed mitochondrial function due to prolonged starvation and also that type III inclusions (crystal or crystalloid) originate from the mitochondrial membrane and matrix.
\end{abstract}

In the past few decades, considerable attention has been paid to intramitochondrial inclusions with respect to mitotochondrial structure and function. However, the mode of formation and especially the fate of these inclusions have been unclear except for those found in frog oocytes (Massover 1971a and b), and rat brown adipocytes (Barnard and Lindberg 1969. Lindgren and Barnard 1972, Suter 1969). The disappearance of inclusions has been reported in rat brown adipocytes in the early hours following birth (Barnard and Lindberg 1969, Lind- gren and Barnard, 1972, Suter 1969) and in rat hepatocytes during rehydration after prolonged water deprivation (Bartok et al 1975). However, there have not been any precise experimental studies known to us on the process of restoration of the unusual mitochondria to more common forms.

In previous work, we reported the occurrence of unusual mitochondria with three types of inclusions in pancreatic acinar cells of the starved Japanese newt (Taira 1979). Type I inclusion is a localized enlargement of the mitochondrial 
matrix or an area devoid of cristae. Type II inclusion is a dense homogeneous body. Type III inclusion is a crystal or crystalloid. Other unusual mitochondria have parallel arrays of cristae. The purpose of this study as an approach to understanding the structure and function of mitochondria is to elucidate more detailed morphological features of these unusual mitochondria which are restored to common forms after re-feeding without any degenerative changes.

\section{Materials and Methods}

Japanese newts, Triturus pyrrogaster, of both sexes were collected in the suburbs of Maebashi in the spring, and adapted to the laboratory aquarium at $17^{\circ} \mathrm{C}$ for one month being fed commercially obtained swine liver regularly every 3 days. Thereafter the animals were starved for 4 weeks (control), then groups comprising five newts each were killed at 3, 6 and 12 hours, and at 1,2, 4 and 8 days after the first re-feeding. Feedings were successively carried out at 3 and 6 days after the first re-feeding. Preparations of small pancreatic tissues for electron microscopic examination were similar to those described in our previous study (Taira 1979).

\section{Results}

Three marked changes were observed in pancreatic acinar cells of the starved animals. Namely, many fat droplets were seen in the basal portion of the cytoplasm, there were unusual types of mitochondria (Figs. 1-3) and vesicular or whorled endoplasmic reticulum (ER).

Zymogen granules decreased in number in the early hours following re-feeding and thereafter they increased quickly. Marked formation of zymogen granules was found in animals on the 2nd day following re-feeding. The profiles of ER changed from vesicular to tubulo-vesicular forms at 3 hours following re-feeding. Whorls of ER slowly decreased in number and size but were occasionally found in the perinuclear zone even on the 8th day following re-feeding (Taira 1981). Fat droplets also decreased in number and size. Intracisternal granules and annulate lamellae were found in several cells.

As for mitochondrial changes, intramitochondial granules gradually decrcased in number. In early stages following refeeding clusters of mitochondria were frequently observed (Figs. 4-6). They were located close to each other and smooth-surfaced cisternae of ER were interposed between these mitochondria (Fig. 4). Thereafter these contacting mitochondria decreased in number. No other unusual mitochondria, different from those observed in the previous study (Taira 1979), were seen in the acinar calls of re-fed animals. The situation which restores unusual mitochondria to common forms varied from cell to cell; since all the mitochondria did not change synchronously and the mitochondria in all the cells did not show similar structures at the same stage after refeeding. In the process of restoration in clusions were only found in the mitochondrial matrix, and degenerating mitochodria were never found in any observations in the present experiment.

Type I inclusions (Fig. 5) were found from 3 hours to one day following refeeding, and thereafter decreased in number. They disappeared more quickly than type III inclusions or parallel arrays of cristae. Their enlarged matrix area was filled with a large amount of amorphous or fine granular substance similar to that found in the matrix of common mitochondria. Type II inclusions (Fig. 7) also disappeared by the 4 th day following re-feeding, but their precise process of 
recovery to common mitochondria was not demonstrated in the present experiment, since they were not numerous and were difficult to evaluate. Type III crystal inclusions became small and their latticelike parallel pattern appeared indistinct following re-feeding (Fig. 8). Type III crystalloid inclusions also became small and irregular, and parts looked quite similar to their inner membrane or cristae (Figs. 9 and 10). Mitochondria with parallel arrays of cristae became smaller and shorter than those observed in the starved animals and the frequency of mitochondrial cristae aligned obliquely in their long axis increased (Fig. 11). Some mitochondria had wavy and irregular cristae inclined at various angles to their long axis. By the 2nd day following re-feeding none of these parallel arrays of cristae were found in any mitochondria examined.

\section{Discussion}

The present study confirmed the findings of our previous work (Taira 1979) that three types of intramitochondrial inclusions were located only in the mitochondrial matrix. They may be induced during prolonged starvation and restored to common forms following re-feeding. No evidence was found to support the hypothesis that mitochondria with various inclusions degenerate. The restoration from unusual mitochondria to common forms has been reported in a few types of inclusions of rat brown adipocytes (Barnard and Lindberg 1969, Lindgren and Barnard 1972, Suter 1969), hepatocyte Bartok et al 1973) and follicular cell of rabbit thyroid glands (Nathaniel 1978).

In the first few hours following refeeding, some mitochondria were positioned in close proximity to each other. Moreover, their outer membranes were sometimes closely in contact with the elongated part of the ER. According to an earlier report (Bew et al. 1969), clusters of mitochondria have been found in hepatocytes of fasted rats after prolonged water deprivation. In these rats interdigitations of the membranes of adjacent mitochondria often formed chains of mitochondria. There are protrusions of one mitochondria into another. However, the function of these clusters of mitochondria remains to be elucidated.

Close apposition between ER and mitochondria has been reported in oocytes (Kessel 1971), hepatocytes (Bartok et al 1973, Riede and Rohr 1971, Tuchweber et al 1972, Norton et al. 1977) and follicular cells of the thyroid (Nunez et al. 1975). It is difficult to imagine that the close apposition between ER and mitochondria is without functional significance. Recent studies suggest that such mitochondria-ER complexes may operate as functional units in the synthesis an assembly of cytochrome P-450 and possibly in other biosynthetic processes involving these organelles (Meier et al. 1978, Pickett et al. 1981). Our previous and present studies also demonstrated the close apposition between mitochondria and ER. These findings suggest the hypothesis that the raw material of the intramitochondrial inclusion is produced in the ER and transported from the ER into the mitochondria.

Type III crystal inclusions became small after re-feeding. Sometimes the periphery of the crystal inclusion is present in close proximity to the enlarged matrix of the mitochondria. From the previous and present results, it seems reasonable to assume that type III crystal inclusions originate from the mitochondrial matrix.

It is an important finding that type I inclusions were not found in animals starved for 4 weeks but became prominent within the first few hours following refeeding. In the previous work type I inclusions had been found in one or two 
weeke of starvation. Therefore, it seems likely that type I inclusions are a transitional form in the formation of or restoration from type III crystal inclusions. The above-mentioned assumption accords with the findings of mitochondria with enlarged matrix in amphibian oocytes which may be in a temporarily form of intramitochondial yolk formation (Massover 1971a). Type I inclusions look very similar to mitochondria with enlarged matrix seen in amphibian ooytes, tempting us to conclude that they perform an identical function.

On the other hand, type III crystalloid inclusions may originate from mitochondrial cristae or inner membranes, since the continuity between type III crystalloid inclusions and the mitochondrial inner membranes or cristae was sometimes found. Following re-feeding mitochondria with highly ordered structures of type III crystalloid inclusions seemed to diminish representing a continuity of the inclusions with the mitochondrial membrane. It is clear that mitochondria with parallel arrays of cristae are a variation of mitochondria with common transversed cristae.

From the previous and present studies, it seems reasonable to assume that intramitochondrial inclusions in the newt pancreas originate from mitochondrial membranes and matrix. However, the chemical nature of the inclusions remains to be elucidated.

\section{References}

1) Barnard, T. and Lindberg, L.: Ultrastructural changes in the chondriome during perinatal development in brown adipose tissue of rats. Intramitochondrial inclusions. J. Ultrastr. Res., 29 : 293-310, 1971.

2) Bartok, I., Viragh, Sz. and Menyhart, J.: Prompt divisions and peculiar transformation of cristae in liver mitochondria of rats rehydrated after prolonged water deprivation. J. Ultrastr. Res., 44 : 49-51, 1973.

3) Bew, Y.L., Hetenyl, G. and Phillips, M. J.: Mitochondrial myelin-like figures: a non-specific reactive process of mitochondrial phospholipid membranes to several stimuli. Z. Zellforsch., 99 : 491-506, 1969.

4) Kessel, R.G.: Cytodifferentiation in the Rana pipience oocyte. II. Intramitochondrial yolk. Z. Zellforsch., 112: 313-332, 1971.

5) Lindgren, G. and Barnard, T.: Changes in interscapular brown adipose tissue of rat during perinatal and early perinatal development and after cold acclimation. IV. Morphometric investigation of mitochondrial membrane alterations. Exptl. Cell Res., 70: 81-90, 1972.

6) Massover, W. H. : Intramitochondrial yolkcrystals of frog oocytes. I. Formation of yolk-crystal inclusions by mitochondria during bullforg oogenesis. J. Cell. Biol., 48: 266-279, 1971a.

7) Massover, W.H. : Intramitochondrial yolkcrystals of frog oocytes. II. Expulsion of intramitochondrial yolk-crystals to form single-membrane bound hexagonal crystalloids. J. Ultrastr. Res., 36: 603-620, 1971b.

8) Meier, P.J., Spycher, M.A. and Meyer, U. A. : Isolation of a subfraction of rough endoplasmic reticulum closely associated with mitochondria. Exptl. Cell Res., 111 : 479-483, 1978.

9) Nathaniel, D.R.: Effect of gonadectomy on the follicular cell and inclusions. in mitochondria of rabbit thyroid gland. Am. J. Pathol., 91 : 137-148, 1978.

10) Norton, W. N., Daskal, I., Savage, H.E., Seibert, R.A., Busch, H. and Lane, M.: Effects of galactoflavin-induced riboflavin deficiency upon rat hepatic cell ultrastructure. Virchows Arch., 23 : 353-361, 1977.

11) Nunez, E.A., Greif, R.L. and Gershon, M.D.: Paracrystalloids from mitochondria in thyroid follicular cells. Normal occurrence and experimental induction. Lab. Invest., 33 : 352-362, 1975.

12) Pickett, C.B., Rosenstein, N.R. and Jeter, R.L.: The physical association between 
rat liver mitochondria and rough endoplasmic reticulum. II. Possible role RER-MT complexes play in the biosynthesis of cytochrome P-450. Exptl. Cell Res., 132 : 225-234, 1981.

13) Riede, U.N. and Rohr, H.P.: Atypische Lebermitochondrien adaptative Sonderformen? I. Ultrastructurell-morphologische Untersuchung. Virchows Arch., 8 : 350-356, 1971.

14) Suter, E. R.: The fine structure of brown adipose tissue. II. Perinatal development in the rat. Lab. Invest., $21: 246-258$, 1969.

15) Taira, $K$ : Studies on intramitochondrial inclusions in the pancreatic acinar cells of the Japanese newt, Triturus pyrrogaster. I. Occurrence of intramitochondrial inclusions with long-term starvation. J. Ultrastr. Res., 67: 89-94, 1979.

16) Taira, K.: Ultrastructural study of the whorls of rough endoplasmic reticulum in the exocrine pancreatic cells of the starved and re-fed newts. Biomed. Res., 2 : 194-201, 1981.

17) Tuchweber, B., Kovacs, K., KhandekarJ.D. and Garge, B.D.: Intramitochondrial lamellar formations induced by pregnenolone-16- $\alpha$-carbonitrile in the hepatocytes of pregnant rats. J. Ultrastr. Res., 39 : 456-464, 1972. 


\section{Explanation of Figures}

\section{Abbreviation}

W, Whorled ER.

\section{Plate I}

Fig. 1. The enlarged matrix of a type I inclusion (smaller arrow) in a fasted newt consists of a large amount of fine granular substance. The larger arrow indicates a type III inclusion. The cytoplasm of acinar cells contains tubulo-vesicular and whorled ER(W). The bar represents $0.5 \mu$ in all figures. $\times 33,000$. 


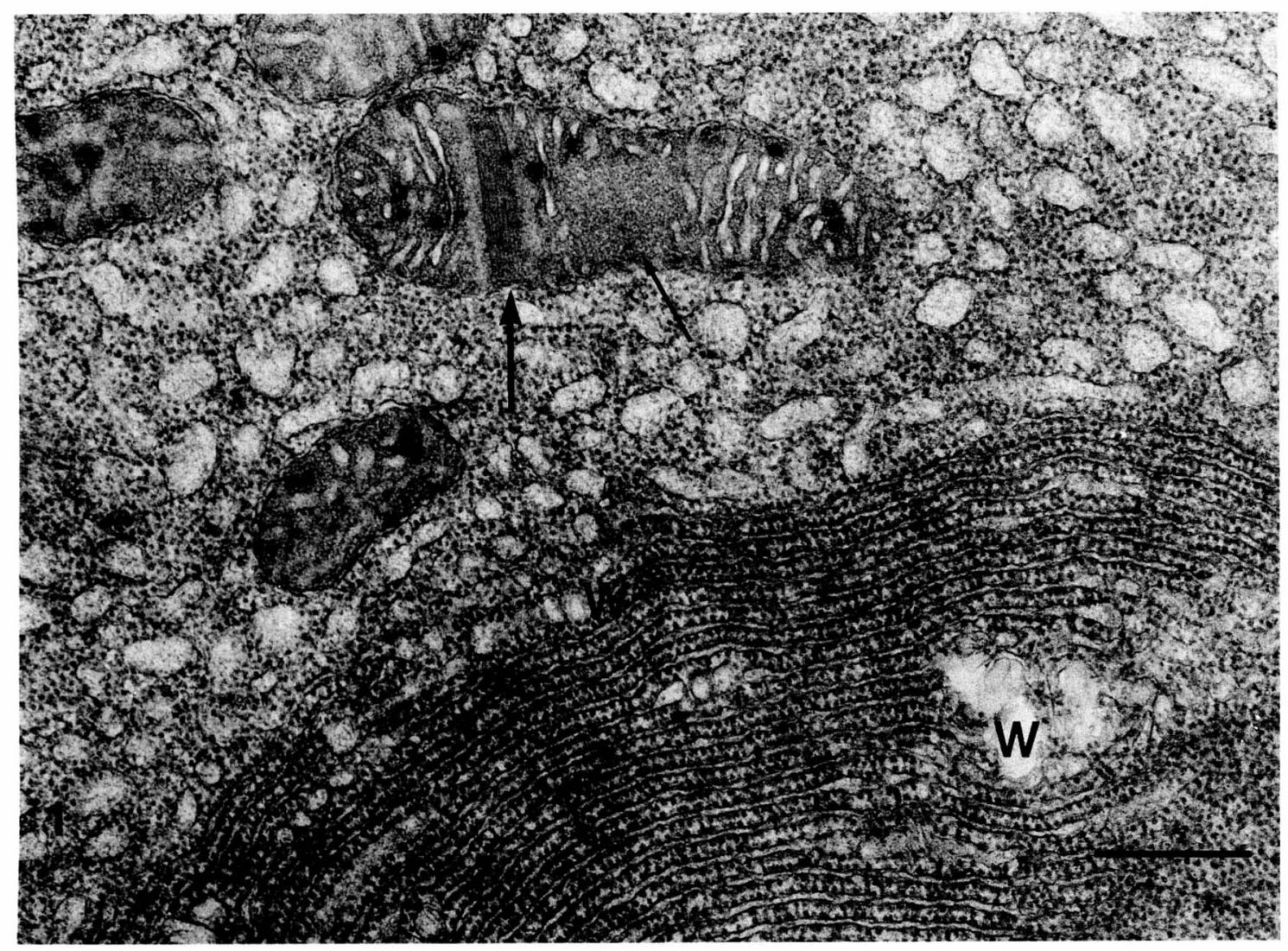

K. Taira and S. Shibasaki 


\section{Plate II}

Fig. 2. A type III crystal inclusion in a fasted newt. It is directly embedded in the matrix without any surrounding membrane or hollow space. The distance between adjacent dense bands is found to be about $130 \AA$ and the dense bands meet at the long axis of the mitochohdrion at an angle of about $60^{\circ} . \times 46,000$.

Fig. 3. A type III crystalloid inclusion in a fasted newt. Highly organized structures are discernible within the matrix. $\times 93,000$. 
Plate II
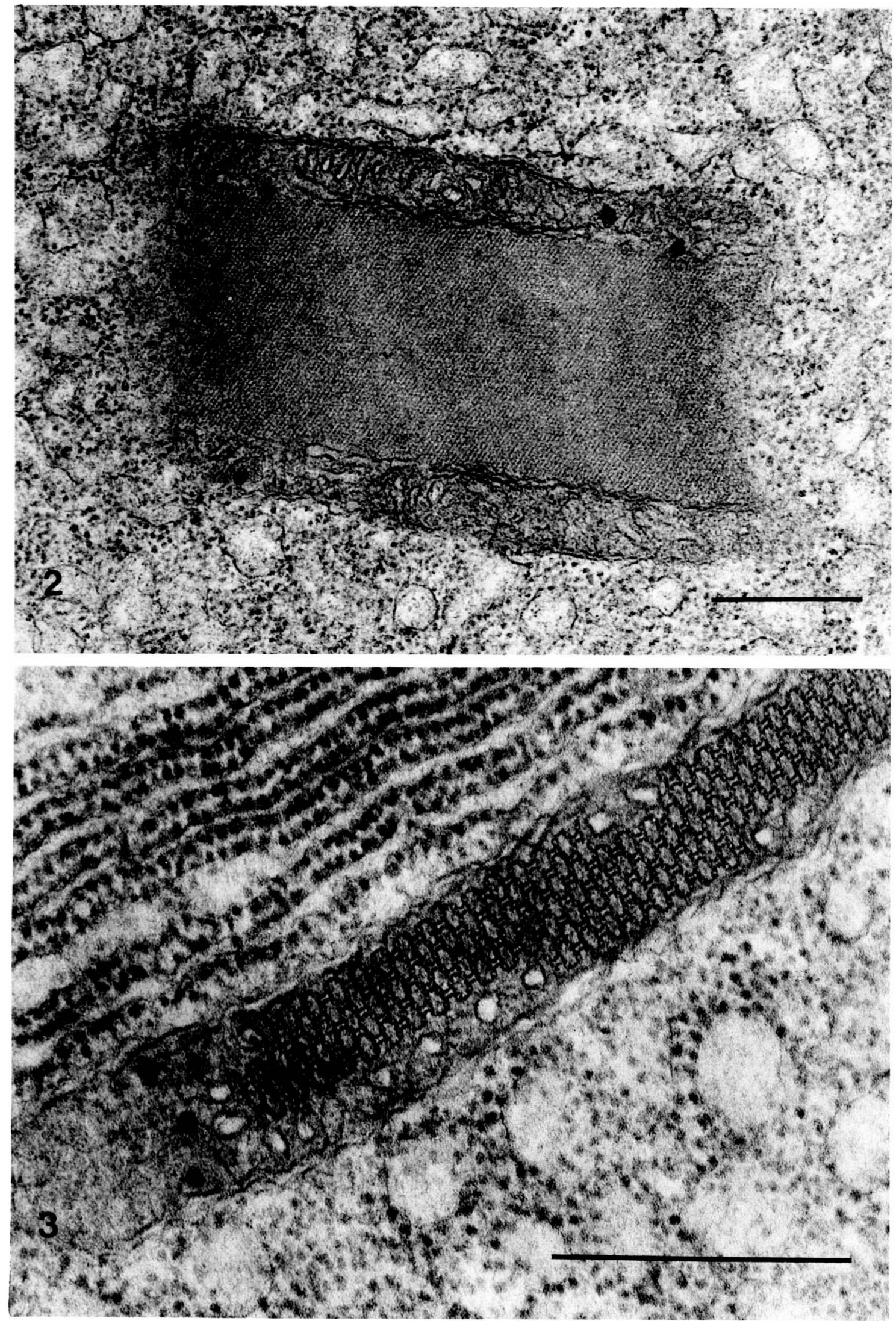

K. Taira and S. Shibasaki 


\section{Plate III}

Fig. 4. The close apposition of mitochondria and ER in a re-fed newt (6 hours following refeeding). Part of the ER degranulates (arrow) and seems to be sandwiched by closely locating mitochondria. $\times 36,000$.

Fig. 5. Four mitochondria in close contact with each other in a re-fed newt (6 hours following re-feeding). One contains an enlarged matrix (type I inclusion). $\times 36,000$.

Fig. 6. Parallel arrays of cristae membranes (arrow) are found in one clustered mitochondria (6 hours after re-feeding). $\times 42,000$.

Fig. 7. Mitochondrion containing dense osmiophilic bodies (type II inclusions) in a re-fed newt ( 2 days following re-feeding). The degradation process in this type of inclusion is obscure because of their scarcity. $\times 31,000$. 
Plate III
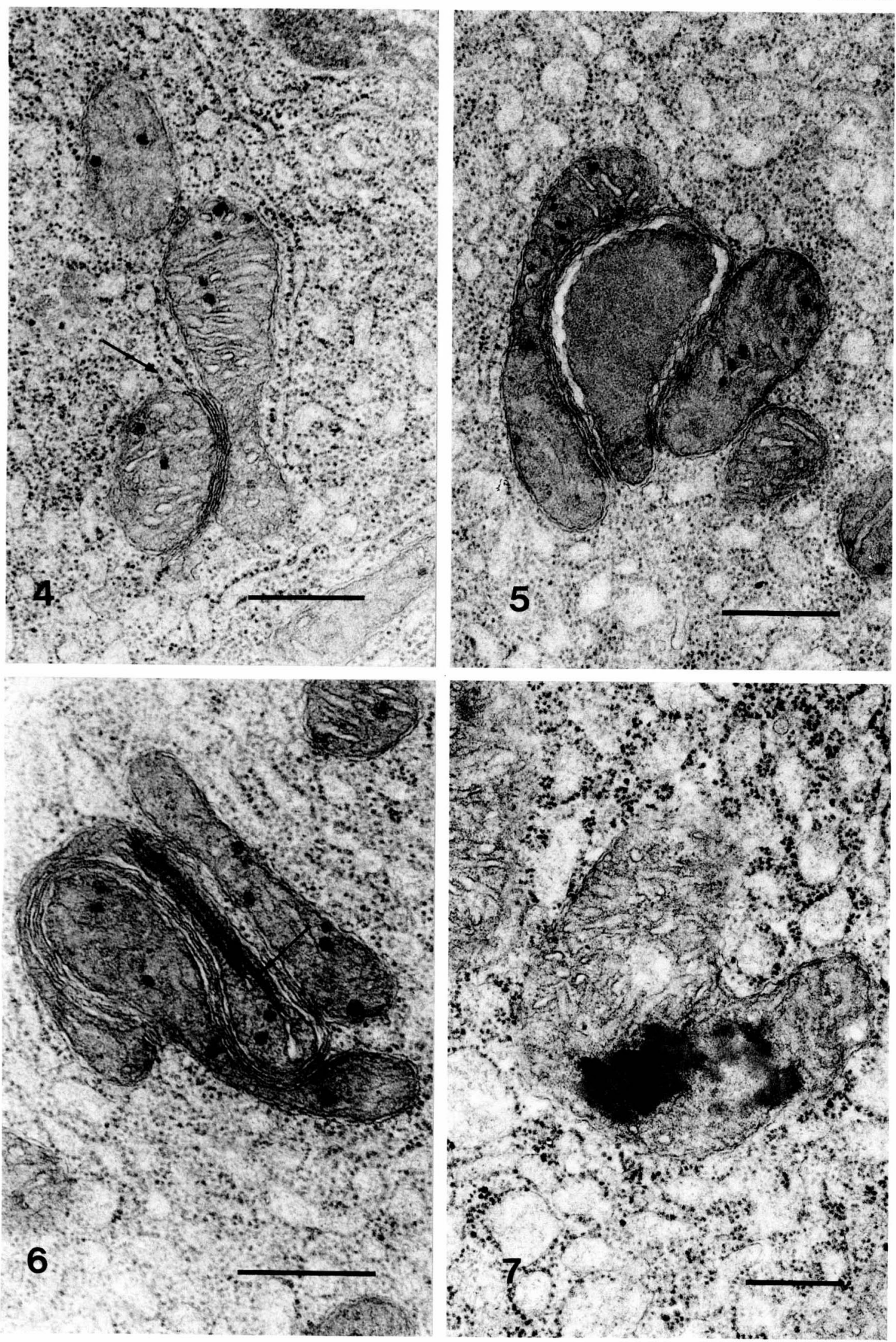

K. Taira and S. Shibasaki 


\section{Plate IV}

Fig. 8. The coexistence of type I (smaller arrow) and type III crystal (larger arrows) inclusions in a mitochondrion of a re-fed newt ( 6 hours following re-feeding). An indistinct parallel pattern seems to represent the residual of type III crystal inclusions. Note the transversely and longitudinally arranged cristae in the mitochondrion. $\times 50,000$.

Fig. 9. The membranous structures of type IIII crystalloid inclusions closely resemble mitochondrial cristae (2 days after re-feeding). $\times 45,000$.

Fig. 10. Residual structures (arrow) may represent a transition from type III crystalloid inclusions to common cristae ( 3 hours following re-feeding). $\times 44,000$.

Fig. 11. A mitochondrion contains cristae which meet at various angles along their long axis (2 days afterire-feeding). The smaller arrows indicate the faint parallel structures of type III crystal residues. The larger arrow indicates the continuity between the ordinary cristae and dense parallel arrays. $\times 40,000$. 
Plate IV
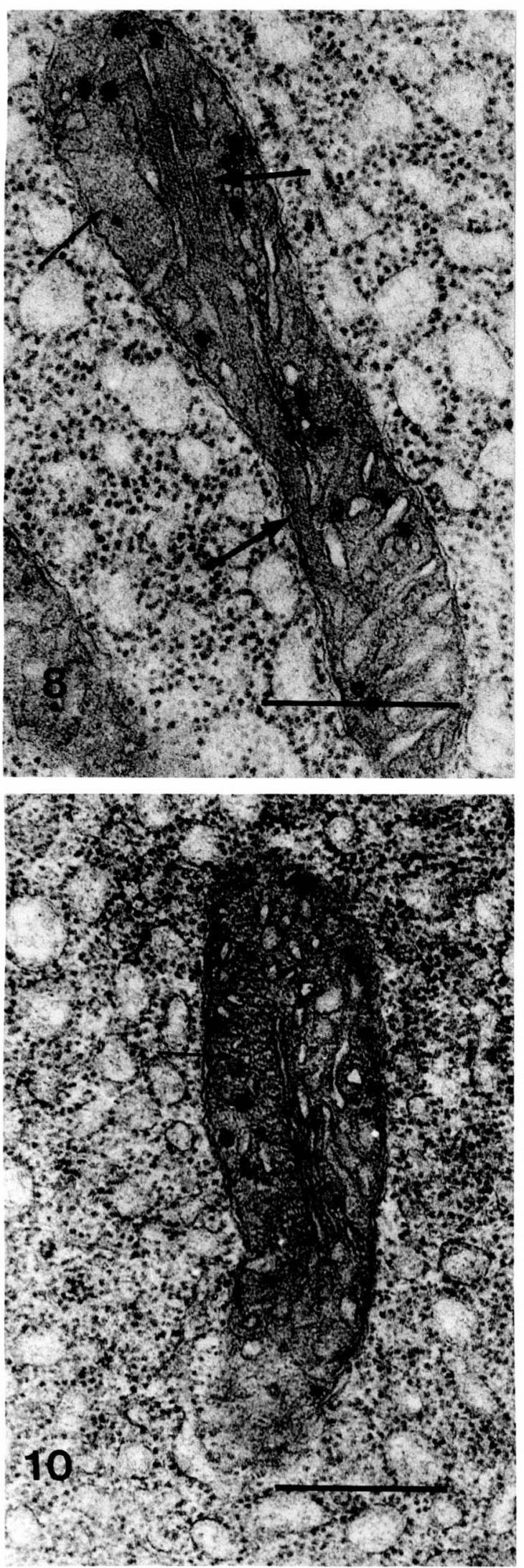

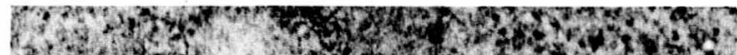

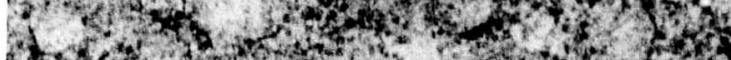

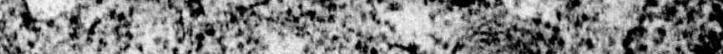

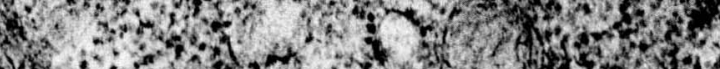

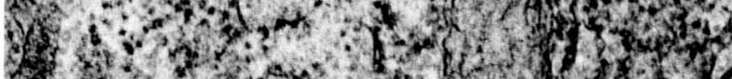

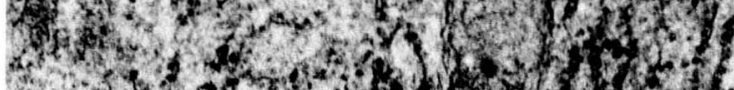

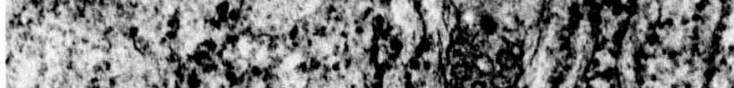

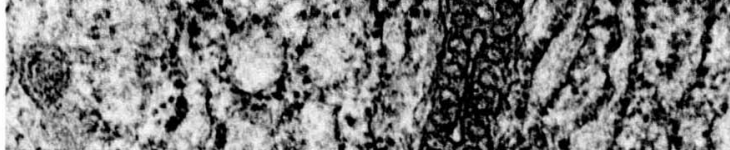

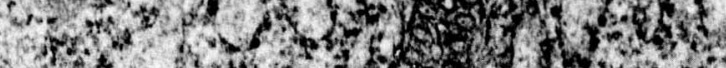
It

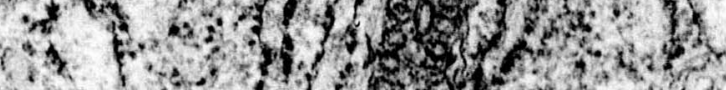
(5) - in

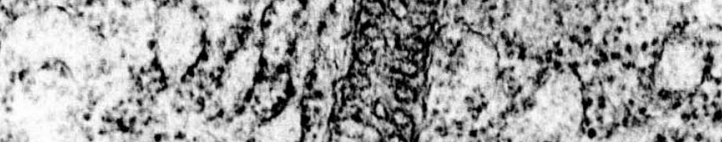

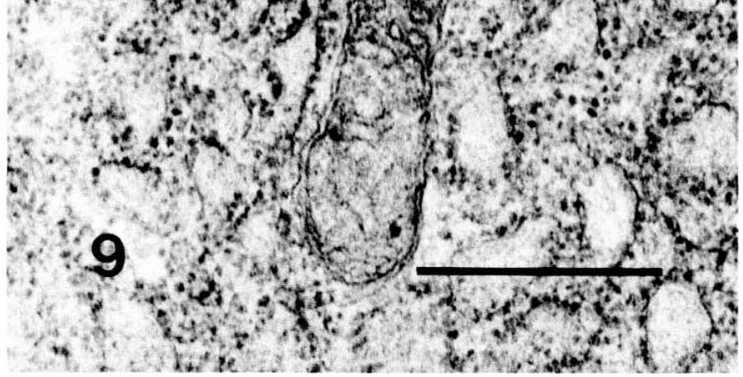

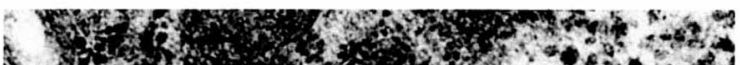

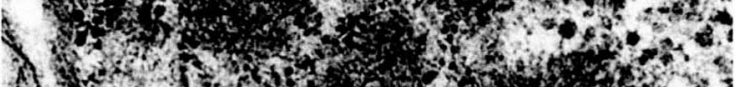

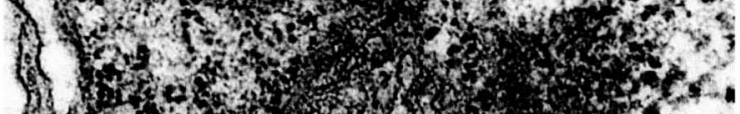

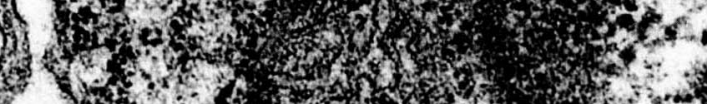

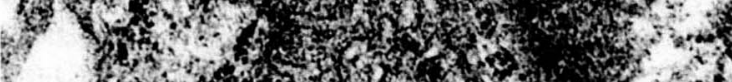

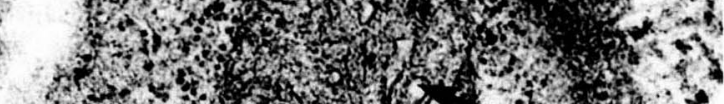

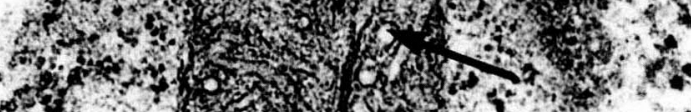
ans

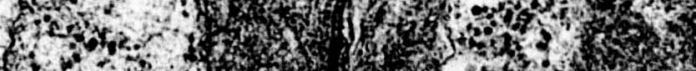

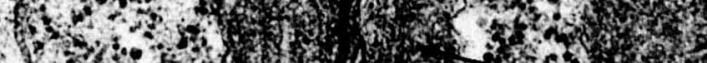

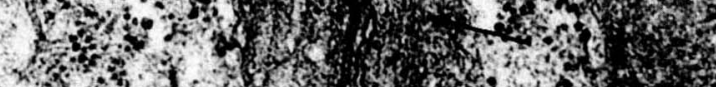
n.t.

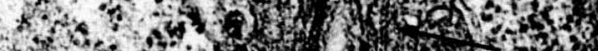

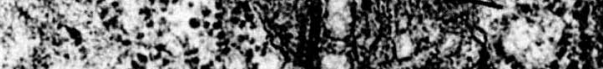

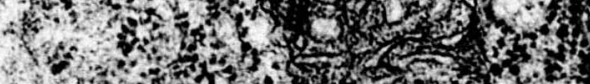
ant 320 on

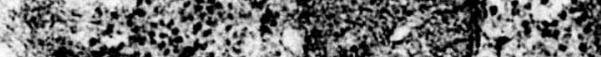

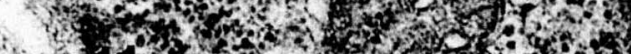

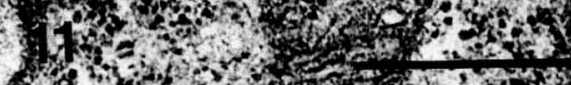

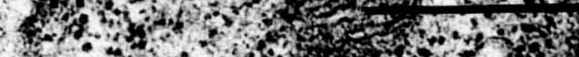

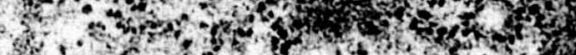

K. Taira and S. Shibasaki 